\title{
Clinical evaluation of a paper chart for predicting ruthenium plaque placement in relation to choroidal melanoma
}

\begin{abstract}
Purpose To determine the accuracy of estimated tumour location and required plaque position using fundus diagrams, and to evaluate their applicability in daily clinical practice.

Patients and methods Between September 2013 and March 2016, all patients treated with ruthenium plaque brachytherapy for choroidal melanoma at the Department of Ophthalmology in Poznań underwent pretreatment planning with the use of printed or electronic fundus diagrams (www. oculonco.com). The estimated distances were then verified intraoperatively.

Results There were 40 eyes of 40 patients: 15 men, 25 women, with a median age of 61 years (range: 21-88). The median longitudinal basal diameter (LBD) of the treated melanomas was $10.4 \mathrm{~mm}$ (range: $6.5-14.9$ ) and the median thickness was $4.1 \mathrm{~mm}$ (range: 1.9-6). The final postition of the anterior tumour margin was within $1 \mathrm{~mm}$ of the estimated location in 39 cases $(97 \%)$, and within $2 \mathrm{~mm}$ in 1 case ( $3 \%$ ). Median followup was 15 months (range: 3-36). By the close of the study, there was one local tumour recurrence $(3 \%)$ and one patient died of unrelated cause 1 year after treatment. No patient developed metastases by study close. Conclusions The plaque planning system using the paper fundus diagrams proved to be accurate, easily applicable and should aid plaque placement where computer modelling

being used most commonly in Europe. The conventional approach is to position the plaque so that it physically overlaps the entire tumour margin by at least $2 \mathrm{~mm}$; however, with posterior tumours, this can cause collateral damage to optic disc and/or fovea. Damato and associates ${ }^{1}$ have therefore developed techniques for positioning the ruthenium plaque with its posterior edge aligned with the posterior tumour margin, relying on side-scatter to provide a safety margin. This requires accurate plaque placement in relation to the tumour.

The aim of this study was to determine whether choroidal melanomas could be accurately localised by drawing fundus diagrams using a novel diagram as an aid to plan ruthenium plaque insertion.

\section{Material and methods}

From September 2013 to March 2016, all patients treated with ruthenium plaque for uveal melanoma at the Department of Ophthalmology, Poznań University of Medical Sciences, Poland, underwent pretreatment planning using fundus diagrams (designed by BD), which were downloaded from www.oculonco.com. All procedures were performed using the $20 \mathrm{~mm}$ CCB ruthenium plaque (Bebig, Berlin, Germany) according to the standard protocol. The written consent was obtained from all the patients prior to the surgery.
\end{abstract} is not possible.

Eye (2018) 32, 421-425; doi:10.1038/eye.2017.205; published online 29 September 2017

\section{Introduction}

Most choroidal melanomas are treated with brachytherapy, with ruthenium applicators
I Rospond-Kubiak ${ }^{1}$ J Kociecki ${ }^{1}$ and B Damato ${ }^{2,3}$

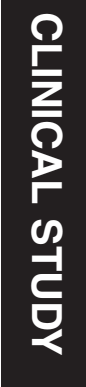

\section{Pre-operative assessment}

The diagnosis of uveal melanoma was established on the basis of clinical examination, colour fundus photographs, autofluorescence (Topcon 50DX IA, Tokio, Japan), and ultrasound (Ellex EyeCubed, Sydney, NSW, Australia).
${ }^{1}$ Ocular Oncology Service, Department of Ophthalmology, Poznań University of Medical Sciences, Poznań, Poland

${ }^{2}$ Ocular Oncology Service, Department of

Ophthalmology, University of California,

San Francisco, CA, USA

${ }^{3}$ Department of Radiation Oncology, University of California, San Francisco, CA, USA

Correspondence: Dr I Rospond-Kubiak, Department of Ophthalmology, Poznań University of Medical Sciences, 1/2, Długa Street, Poznań 61-848, Poland Tel: +48 618549262; Fax: +48 618549084 E-mail: iwona.rospondkubiak@wp.pl

Received: 19 March 2017 Accepted in revised form: 17 July 2017 Published online: 29 September 2017 
In every case, we measured the longitudinal basal diameter (LBD) and transverse diameter of the tumour, and the distance between the tumour and the optic disc.

\section{Planning procedure}

The fundus diagram consisted of concentric circles, indicating $1 \mathrm{~mm}$ intervals, and radial lines in clock minutes (Figure 1). The procedure of planning was as follows: first, the meridians of the lateral tumour margins were marked on the chart by superimposing the fundus photographs on the chart and the main meridian in which the plaque needed to be placed was established. Next, the location of the posterior tumour margin was drawn, using measurements from ultrasonography and/or fundus photographs (ie, the distance between the optic disc and the the tumour margin). Then, the location of the anterior tumour margin was identified, using measurements from ultrasonography, and marked on the diagram. The limbus location was estimated if there was a refractive error, using the axial length and a table and marked on the chart. Finally, the required position of the plaque (ie, $2 \mathrm{~mm}$ margins or eccentric) was established.

\section{Surgical technique}

The surgical technique of plaque insertion has been described previously. ${ }^{2}$ All procedures were performed under general anaesthesia. The extraocular muscles were dis-inserted, if necessary, to ensure good visibility of the tumour base. The tumour was localised by trans-ocular transillumination, which was performed with a 20G fibreoptic vitrectomy light pipe pre-bent to $90^{\circ}$, with its tip placed on the sclera on the other side of the globe (eg, in the medial equatorial region if the tumour was located in the temporal, equatorial choroid). Next, an ink-mark was made on the sclera at the anterior tumour margin, half-way between the two lateral tumour margins. The desired location of the anterior plaque edge was estimated by subtracting the longitudinal tumour diameter from the plaque diameter and marked on the sclera with a pen. A transparent template (dummy plaque) was sutured to the sclera with its anterior edge aligned with the scleral ink-mark, using releaseable knots placed in two lugs at the antero-lateral edges of the template. The sutures were placed circumferentially (parallel to the limbus). The location of the template in relation to the tumour was assessed by placing the tip of the transilluminate at the edge of the template while performing binocular indirect ophthalmoscopy. If the template needed re-positioning, it was pulled or pushed with the transilluminator into its correct location, while performing the ophthalmoscopy, and an ink-mark was made on the sclera at the template's anterior edge, halfway in between the two lugs. Once the template was correctly positioned, the lug sutures were untied and the

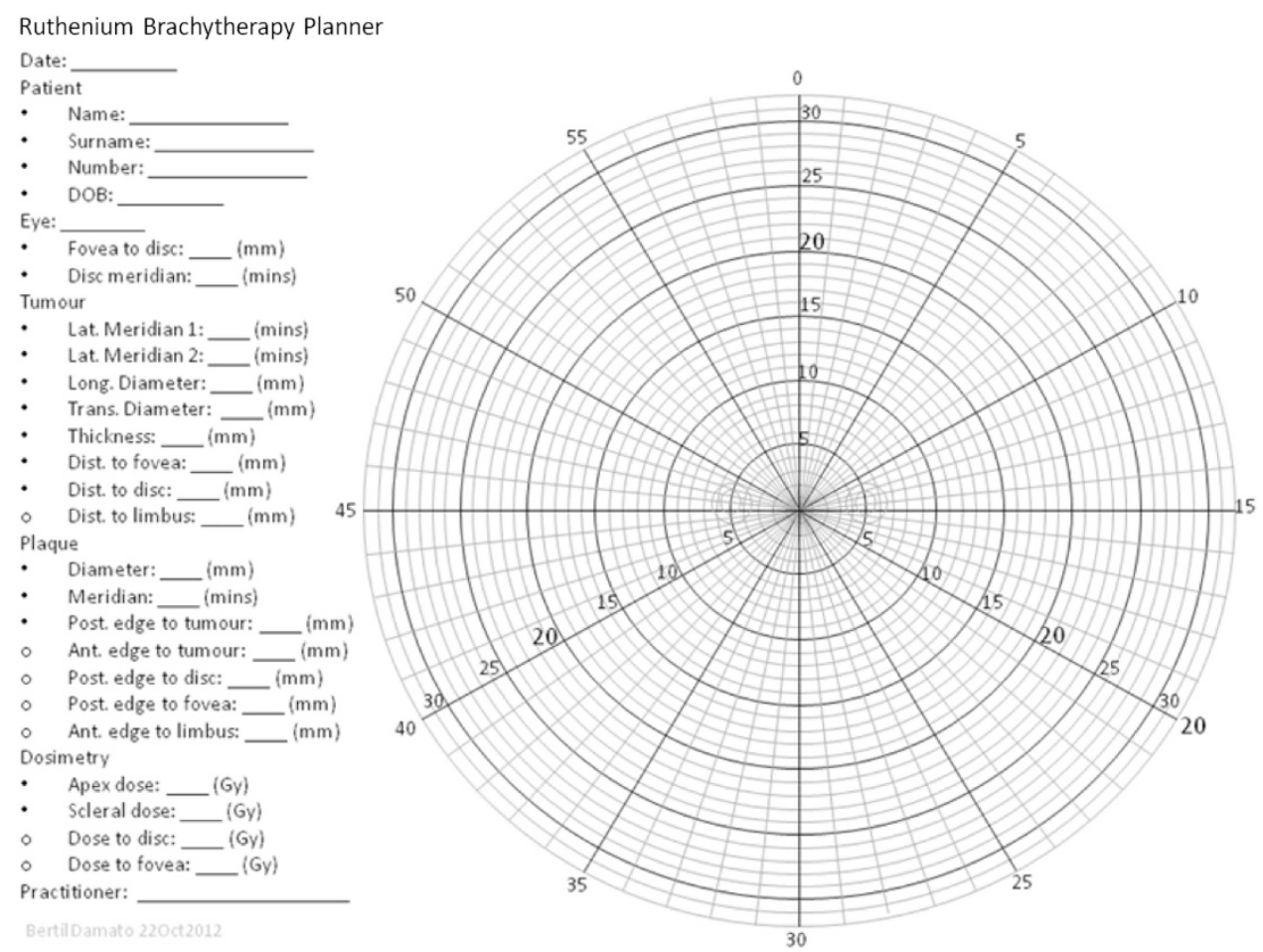

Figure 1 The fundus diagram (internal eye) used for estimating the tumour location and intended plaque position. Additional drawnings can also be downloaded free of charge from www.oculonco.com. 
radioactive plaque was inserted, securing it in place by means of the lug sutures. If there was a need to position the plaque further posteriorly by $1 \mathrm{~mm}$, the lug sutures were tied anteriorly on the lugs without removing them from the sclera; if the plaque needed to be placed more anteriorly, then the sutures were shifted to the posterior rim of the lugs and secured longitudinally. Finally, a mattress suture was placed to ensure that the plaque was firmly apposed to the scleral surface.

For the purposes of this study, the tumour-to-limbus distance was measured without the surgeon referring to the estimated pre-operative tumour-to-limbus distance. The predicted and measured distances were then compared.

The study was conducted in accordance with the Declaration of Helsinki. Ethical committee approval was applied for but not required as this was an evaluation of accepted clinical practice.

\section{Results}

The cohort consisted of 40 consecutive patients (15 male and 25 female) with a mean age of 61 years (range: $21-88)$. The tumour was located in the left eye in 19 (48\%) patients and the right eye in 21 (52\%). The longitudinal basal tumour diameter averaged $10.4 \mathrm{~mm}$ (range: 6.514.9) and the tumour thickness had a median of $4.1 \mathrm{~mm}$ (range: 1.9-5.9) (Table 1). All tumours but one extended posterior to the equator, with $13(33 \%)$ extending to within $5 \mathrm{~mm}$ of the optic disc and $16(40 \%)$ to within $3 \mathrm{~mm}$ of the fovea.

The location of the anterior tumour margin that was estimated pre-operatively was identical to the tumour localised by transillumination in 27 cases (68\%), within $1 \mathrm{~mm}$ in 12 cases (30\%) and within $2 \mathrm{~mm}$ in 1 patient $(2 \%)$.

The plaque position that was estimated pre-operatively was the same as the actual plaque position in 27 patients $(68 \%)$, within $1 \mathrm{~mm}$ in 11 patients $(28 \%)$, and within $2 \mathrm{~mm}$ in two patients (5\%). Figure 2 shows the predicted $v$ s the measured tumour-to-limbus distances. In one of these two patients, the eccentric plaque placement was abandoned because the tumour was located more anteriorly than we had anticipated. In the second of these cases, severe retinal detachment precluded accurate transillumination.

The follow-up had a median of 15.5 months (range: 3-26). By the close of the study, tumour recurrence developed at the posterior margin of a $10.6 \mathrm{~mm} \times 9.2 \mathrm{~mm} \times 3.2 \mathrm{~mm}$ tumour 13 months postoperatively; this was treated by transpupillary thermotherapy.
No patient developed post-operative diplopia. No patient developed metastases by the study close. One patient died of cardiac arrest one year after treatment.

\section{Discussion}

We found that our protocol for predicting plaque location was accurate to within $1 \mathrm{~mm}$ in 38 (95\%) of patients.

Shah et $a l^{3}$ have estimated that a surgeon needs to insert more than 1000 plaques to achieve the skill needed to consistently place plaques accurately in relation to the tumour. Given the rarity of uveal melanomas, many surgeons would have difficulty achieving this number.

Any measures that make it easier to position radioactive plaque accurately should reduce the learning curve, thereby improving results. Computer programs have been developed to create 3D models of the eye and tumour. ${ }^{4}$ Our paper fundus diagram is therefore intended for situations where such programs are not available.

We have prepared a table to adjust for axial length; however, our results suggest that errors are minimal if the axial length is $12-26 \mathrm{~mm}$.

Several authors employed a variety of techniques for checking the correct plaque position, including intraoperative ultrasound, ${ }^{5}$ magnetic resonance imaging, ${ }^{6}$ plaque-mounted diode-lights, ${ }^{7}$ modified dummy plaques with openings for transilluminator, ${ }^{2}$ and opaque templates. ${ }^{8}$ These can complement the methods described in this article. We performed trans-scleral transillumination with binocular indirect ophthalmoscopy to confirm accurate placement of the template.

Pre-operative tumour localisation allows the surgeon to plan the surgical procedure and to anticipate the intraoperative findings. Pre-operative estimates could also alert the surgeon to any misleading transillumination, thereby encouraging special measures to localise the anterior tumour margin (eg, performing binocular indirect ophthalmoscopy with the transilluminator tip on the sclera at the anterior tumour margin). Pre-operative tumour localisation could also be useful in the rare cases when ophthalmoscopy is prevented by opaque media and when transillimination is inconclusive (eg, if the tumour is amelanotic or if the choroid is hyper-pigmented, as in congenital ocular melanocytosis).

In conclusion, tumour-to-limbus distance can accurately be predicted using our fundus diagram thereby enabling plaque insertion to be planned preoperatively, enhancing accuracy. 
Table 1 Characteristics of the studied group

\begin{tabular}{|c|c|c|c|c|c|c|c|c|c|c|}
\hline No & Sex & Age & Laterality & $L B D$ & $T D$ & $T h$ & Tumour-to-limbus predicted & Tumour-to-limbus measured & Difference & $A L$ \\
\hline 1 & $\mathrm{~F}$ & 39 & Left & 7,4 & 7 & 1,9 & 9 & 9 & 0 & 29,37 \\
\hline 2 & M & 41 & Left & 10,6 & 9,2 & 3,2 & 20 & 20 & 0 & 24,58 \\
\hline 3 & $\mathrm{~F}$ & 79 & Right & 8,8 & 7,7 & 3,1 & 20 & 20 & 0 & 22,17 \\
\hline 4 & $\mathrm{~F}$ & 69 & Right & 10,8 & 9,92 & 3,6 & 12 & 12 & 0 & 22,91 \\
\hline 5 & $\mathrm{~F}$ & 61 & Right & 11,8 & 11 & 4,9 & 20 & 19 & 1 & 20,67 \\
\hline 6 & $\mathrm{~F}$ & 54 & Left & 10,8 & 9,9 & 5,8 & 16 & 16 & 0 & 21,98 \\
\hline 7 & $\mathrm{~F}$ & 75 & Right & 9 & 7,8 & 3,1 & 20 & 20 & 0 & 21,93 \\
\hline 8 & $\mathrm{~F}$ & 61 & Left & 7,9 & 7,5 & 2,6 & 14 & 14 & 0 & 22,8 \\
\hline 9 & $\mathrm{~F}$ & 67 & Right & 15 & 12,8 & 5,1 & 14 & 14 & 0 & 22,84 \\
\hline 10 & $\mathrm{~F}$ & 76 & Left & 10,5 & 10,2 & 3,3 & 16 & 16 & 0 & 23,31 \\
\hline 11 & M & 73 & Left & 8,8 & 6,35 & 2,92 & 7 & 7 & 0 & 22,9 \\
\hline 12 & M & 73 & Left & 10,2 & 9,7 & 4,4 & 19 & 19 & 0 & 23,4 \\
\hline 13 & $\mathrm{~F}$ & 56 & Left & 14,9 & 14,6 & 5,7 & 14 & 12 & 2 & 23,25 \\
\hline 14 & $\mathrm{~F}$ & 21 & Right & 8 & 8,9 & 2,5 & 20 & 20 & 0 & 23,01 \\
\hline 15 & $\mathrm{M}$ & 68 & Right & 7,8 & 7,2 & 2,7 & 15 & 15 & 0 & 24,04 \\
\hline 16 & $\mathrm{M}$ & 61 & Left & 8,8 & 8,1 & 4,7 & 21 & 20 & 1 & 20,35 \\
\hline 17 & M & 53 & Left & 13,7 & 12,4 & 5,6 & 4 & 4 & 0 & 24,5 \\
\hline 18 & $\mathrm{~F}$ & 65 & Right & 13,2 & 10,8 & 6 & 11 & 11 & 0 & 23,58 \\
\hline 19 & $\mathrm{M}$ & 41 & Right & 8 & 7,7 & 3,2 & 16 & 16 & 0 & 23,53 \\
\hline 20 & $\mathrm{M}$ & 82 & Right & 12,4 & 12 & 5,93 & 12,5 & 12,5 & 0 & 23,68 \\
\hline 21 & $\mathrm{~F}$ & 71 & Right & 12,7 & 11 & 6 & 12 & 12 & 0 & 23,66 \\
\hline 22 & $\mathrm{M}$ & 69 & Left & 9,2 & 8,5 & 6 & 7 & 7 & 0 & 22,99 \\
\hline 23 & $\mathrm{~F}$ & 44 & Right & 14,1 & 13,8 & 5,2 & 5 & 5 & 0 & 21,82 \\
\hline 24 & $\mathrm{~F}$ & 70 & Right & 10,3 & 10,3 & 4,7 & 17 & 16 & 1 & 22,49 \\
\hline 25 & M & 41 & Right & 6,5 & 6,72 & 2,5 & 18 & 18 & 0 & 23,18 \\
\hline 26 & $\mathrm{~F}$ & 57 & Left & 10,60 & 13,4 & 4,3 & 12,5 & 13 & 0,5 & 20,84 \\
\hline 27 & $\mathrm{M}$ & 60 & Left & 11,5 & 8,7 & 4 & 17 & 16 & 1 & 23,89 \\
\hline 28 & $\mathrm{~F}$ & 69 & Right & 7,9 & 7 & 2,3 & 16 & 15 & 1 & 21,98 \\
\hline 29 & $\mathrm{~F}$ & 78 & Left & 13,1 & 12,1 & 5,1 & 13 & 13,5 & 0,5 & 22,64 \\
\hline 30 & $\mathrm{~F}$ & 66 & Left & 10,7 & 10,4 & 3,6 & 5,5 & 5,5 & 0 & 22,83 \\
\hline 31 & $\mathrm{~F}$ & 51 & Right & 11,5 & 10,4 & 3,78 & 11 & 11 & 0 & 24,76 \\
\hline 32 & $\mathrm{M}$ & 75 & Right & 12,7 & 14 & 3,4 & 1,5 & 1,5 & 0 & 24,64 \\
\hline 33 & $\mathrm{M}$ & 63 & Right & 12,8 & 12,4 & 4,3 & 6,5 & 7 & 0,5 & 22,48 \\
\hline 34 & $\mathrm{~F}$ & 88 & Right & 8,75 & 8,2 & 3 & 12,5 & 12 & 0,5 & 21,97 \\
\hline 35 & $\mathrm{~F}$ & 49 & Left & 12,60 & 10 & 4,2 & 8 & 8 & 0 & 21,82 \\
\hline 36 & $\mathrm{M}$ & 59 & Right & 9 & 8,8 & 3 & 21 & 20 & 1 & 22,68 \\
\hline 37 & $\mathrm{M}$ & 53 & Left & 8,5 & 10,5 & 4,4 & 11,5 & 11,5 & 0 & 24,57 \\
\hline 38 & $\mathrm{~F}$ & 56 & Right & 7,2 & 7,1 & 4,2 & 17,5 & 17,5 & 0 & 23,4 \\
\hline 39 & $\mathrm{~F}$ & 65 & Left & 10,2 & 9,9 & 4,4 & 16,5 & 17,5 & 1 & 23,1 \\
\hline 40 & $\mathrm{~F}$ & 63 & Left & 9,75 & 8,8 & 3,2 & 15,5 & 16 & 0,5 & 23,4 \\
\hline
\end{tabular}

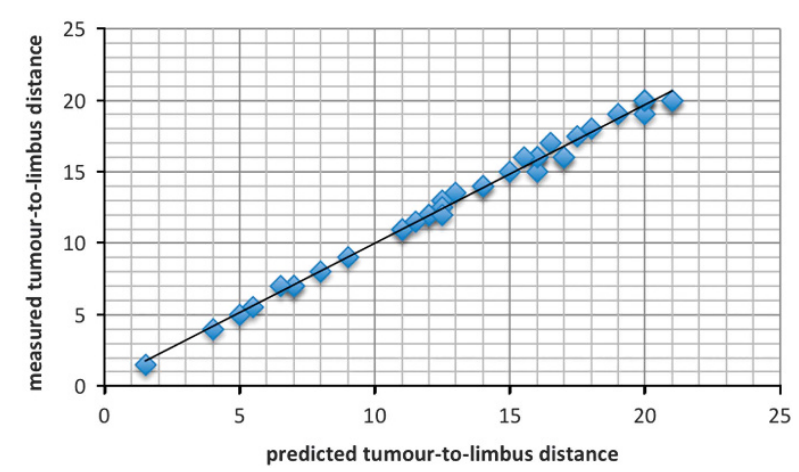

Figure 2 Histogram comparing the estimated tumour-to-limbus distance with measurements taken intraoperatively $(n=39)$.

\section{Summary}

What was known before

- 3D computer modelling is the only system that can be used for estimating plaque position in uveal melanoma treatment.

What this study adds

- Specially designed paper chart can accurately predict the location of anterior tumour margin and estimate the required ruthenium plaque position with relation to choroidal melanoma if computer system is not available.

\section{Conflict of interest}

The authors declare no conflict of interest. 


\section{References}

1 Russo A, Laguardia M, Damato B. Eccentric ruthenium plaque radiotherapy of posterior choroidal melanoma. Graefes Arch Clin Exp Ophthalmol 2012; 250(10): 1533-1540.

2 Seregard S, Damato B. Uveal malignant melanoma: management options for brachytherapy. In: Damato B, Singh AD (eds). Clinical Ophthalmic Oncology: Uveal Tumours. Springer Verlag: Berlin, 2014.

3 Shah NV, Houston SK, Murray TG, Markoe AM. Evaluation of the surgical learning curve for I-125 episcleral plaque placement for the treatment of posterior uveal melanoma: a two decade review. Clin Ophthalmol 2012; 6: 447-452.

4 Berry JL, Dandapani SV, Stevanovic M, Lee TC, Astrahan M, Murphree AL et al. Outcomes of choroidal melanomas treated with eye physics: a 20-year review. JAMA Ophthalmol 2013; 131(11): 1435-1442.
5 Harbour JW, Murray TG, Byrne SF, Hughes JR, Gendron EK, Ehlies FJ et al. Intraoperative echographic localization of iodine 125 episcleral radioactive plaques for posterior uveal melanoma. Retina 1996; 16: 129-134.

6 Hanna SL, Lemmi MA, Langston JW, Fontanesi J, Brooks HL Jr, Gronemeyer S. Treatment of choroidal melanoma: MR imaging in the assessment of radioactive plaque position. Radiology 1990; 176: 851-853.

7 Finger PT, Iezzi R, Esteveo ML, Szechter A, Rosen RB, Berson A. Diode-light transilluminator for ophthalmic plaque localization around juxtapapillary choroidal melanomas. Int $J$ Radiat Oncol Biol Phys 1999; 44: 887-890.

8 Krohn J. A modified dummy plaque for the accurate placement of Ruthenium-106 plaques in brachytherapy of intraocular tumours. Ocul Oncol Pathol 2016;

2: $1-4$. 